\title{
Virus - The Biological Brain Twister
}

\author{
S. Shwetha* \\ Dr. MGR Educational and Research Institute, Maduravoyal, Chennai, Tamil Nadu, India \\ *Corresponding author
}

\begin{tabular}{|c|}
\hline Keywords \\
\hline $\begin{array}{l}\text { Virus, Host cells, } \\
\text { Infections, Microbiology, } \\
\text { Cell biology, Protein } \\
\text { folding, Living entity }\end{array}$ \\
\hline Article Info \\
\hline $\begin{array}{l}\text { Accepted: } \\
04 \text { April } 2018 \\
\text { Available Online: } \\
10 \text { May } 2018\end{array}$ \\
\hline
\end{tabular}

\section{Introduction}

Virus is a small infectious agent that typically consists of nuclei acid molecule in a protein coat. Ever since its time of discovery in the year 1892 by Dmitri Ivanosky, where he described a non-bacterial pathogen infecting the tobacco plants (Fig. 1) till the isolation of this pathogen by Martinus Beijernick and naming of this pathogen called VIRUS (Lecoq et al., 2001), stood out as milestone of Virology history. Though they have made milestones they exhibit the characteristics of both living and non-living (as biochemicals) considered as a microbiological mystery. This entity has constantly sparked debates in the world of science, is it biology or chemistry? Is it alive or dead? Let us analyse about this peculiar question in detail.

\section{Virus - The contradiction}

To declare virus as a living thing, let us see whether virus check all or at least some boxes of lining things.

Life is scientifically defined as organism that,

Maintain Homeostasis

Are composed of cells 
Undergo metabolism

Can grow

Adapt to their environment

Respond to stimuli

Reproduce

So only if virus satisfies these 7 aspects, they can be considered as a living entity. As we are dealing with a very unique organism that are at the verge of life, we surely need to take a closer look or even a different approach in order to give a fair verdict. Hence I am considering certain other factors such as

Evolution

Giant viruses

Protein folding

Along with the already considered seven criteria, let us clear up the point one at a time.

\section{Living organisms maintain homeostasis}

Homeostasis is any self-regulating process by which biological systems tend to maintain stability, while adjusting to conditions that are optimal for survival. Considering viruses are very small even smaller than bacteria (Ventakaraman Prasad et al., 2012). They are just complicated assemblies of biomolecules like protein nuclei acid, lipids and carbohydrates. First of all, a biological system must be made up of cells or at least a cell. Whereas viruses are simply boxes of chemicals (Jake Port, 2017). They neither have the organelles nor have an internal environment for maintaining homeostasis.

Thus virus falls flat when it comes to maintaining Homeostasis conditions.

\section{Living things are composed of cells}

As said earlier living organism must possess cells or a cell. And viruses do not. But they do exhibit complexity in their structure and they do come in different sizes (Ventakaraman Prasad et al., 2012) (ranging from $20 \mathrm{~nm}$ to $1000 \mathrm{~nm}$ ) and exhibit a variety of shapes and symmetry (icosahedral, helical, complex, prolate, spherical) enveloped or nonenveloped virus; classified scientifically as 7 different classes called the BALTIMORE CLASSIFICATION (Fig. 2) depending on the type of genome 1) ds DNA 2) ss DNA 3) ds RNA 4) (+) ss RNA 5) (-) ss RNA 6) ss RNART 7) ds DNA-RT. Though it contains multiform and elaborate and intricate morphology, it is ultimately not a cellule.

Thus viruses nearly miss this criterion.

\section{Living things undergo metabolism}

Anabolism and catabolism together gives metabolism. Thus metabolism is a set of life sustaining chemical transformations within the cells of organism. This is an essential operation to maintain the living state of cells. Viruses are nano particles and agents of infections. They are devoid of ATP and they neither undergo respiration, excretion nor feed on anything.

Hence they fail to hit the mark.

\section{Living things can grow}

Growth and development is a vital quality for any living organism. Viruses are simple entities without any metabolic activity. But they do grow. They aren't capable of independent growth, Eclipse phase in a virus is considered as a phase of growth. Eclipse phase is defined as the time between infection by a bacteriophage or other virus to the time of appearance of mature progeny (Adam et al., 1959). Virus cell therefore is a clear indication of growth and maturation of a viral particle. Virion is usually described as a fully formed entire virus. The growth curve drawn during lytic and lysogenic cycles also imply that 
viruses do grow. However they use and derive energy for their growth from the host.

Thus virus checks this box.

\section{Living things adapt to their environment}

Most viruses are persistent innocuous and non-pathogenic. They remain passive for a long period outside the cell or they take advantage of their host replication machinery to replicate (Villarreal, 2004). As said, their actions exclusively rely upon the environment. They remain as a crystallized inanimate particle, outside and are activated only inside a living host cell for which they are defined as obligate intracellular parasites.

Thus viruses fulfill this necessity.

\section{Living things respond to stimuli}

Viruses are mostly and widely accepted as molecular entities rather than cellular entities. They are organisms that are constantly changing between biology and biochemistry (Villarreal, 2004). As explained earlier viruses do not have any metabolism to provide energy. And they do not contain any energy to respond to any stimuli. Their protective protein coat safeguards them from the harsh temperatures, $\mathrm{pH}$, light or any other cabes that usually stimulates any living organism.

Thus virus fails to satisfy the fact.

\section{Living things reproduce}

Viruses reproduce, to say more accurately they surely do replicate. But they aren't capable of autonomous replication. They do not grow or multiply outside the host instead they hijack the host cells apparatus to carry out their transcriptional and translational processes. Unlike other obligate intracellular parasites such as Chlamydia and Rickettsia that behave as a non-living entity outside the host and use their own replication methods instead of depending on the host's machinery, viruses use host cells DNA or RNA to produce viral proteins. Thus they are self-assembling organic molecules (Patrick Fortterre, 2010). Stuck between the puzzle of replication or reproduction, Claudiu Bandea in 1983 stated that, "In this phase (eclipse phase) the virus shows major physiological properties such as growth, metabolism and reproduction. Therefore life is an effective presence". So whether it is simply an assembly of molecules or the reproduction of daughter cells at the end of lytic and lysogenic cycle, new outputs are surely obtained.

And by this point of view, the reproduction norm is a definite "Yes".

\section{Evolutionary aspect}

Evolutionary and phylogenetic studies have cracked even the most challenging cases in biology. But again, we are met with a dead end as viruses do not fossilize (Viviane Ritcher, 2015). Thus scientists have come up with some possible theories; one theory suggests that both bacteria and virus are descended from a common ancestor. And viruses do share certain macromolecules present in Archae, Bacteria and Eukarya as indicated in Figure 3 (Patrick Fortterre, 2010). All three domains are descended from the Last Universal Common Ancestor (LUCA).

It is also believed that DNA polymerases enzyme present in Eukaryotes have viral origin.

Bacteria evolved to be a more complex form and whereas viruses gradually shed their genes which they found unimportant until they can't even reproduce on their own (Viviane Ritcher, 2015). 
Fig.1 TMV virus

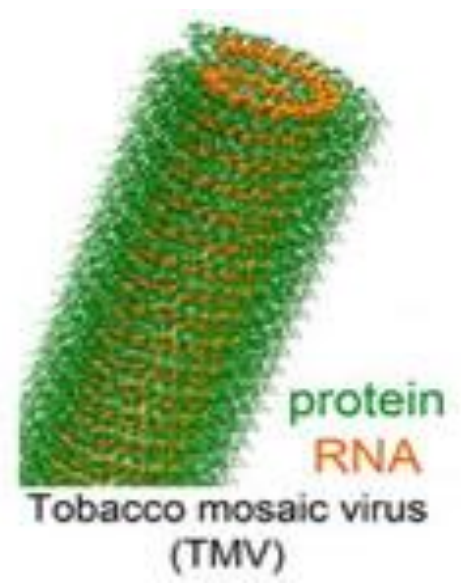

Fig.2 Baltimore classification

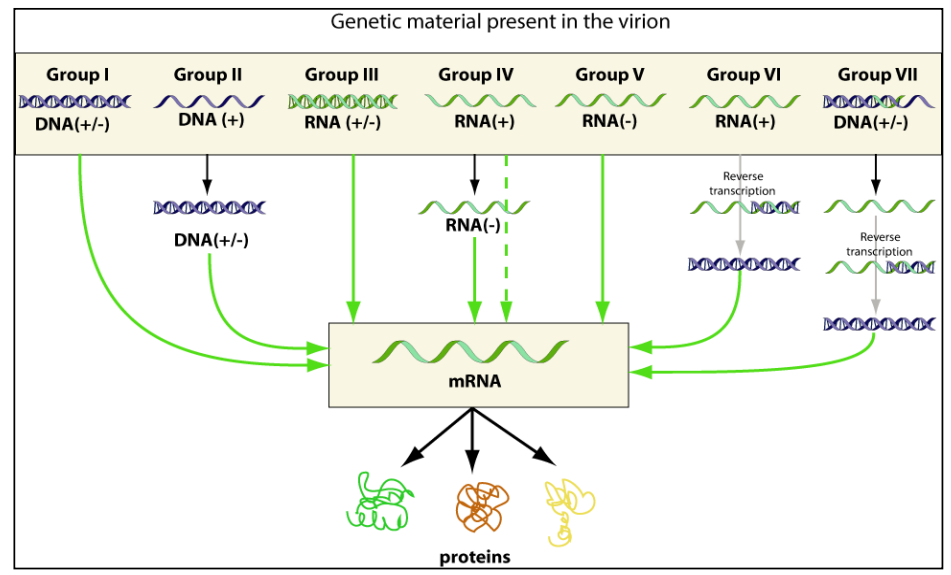

Fig.3 LUCA domain

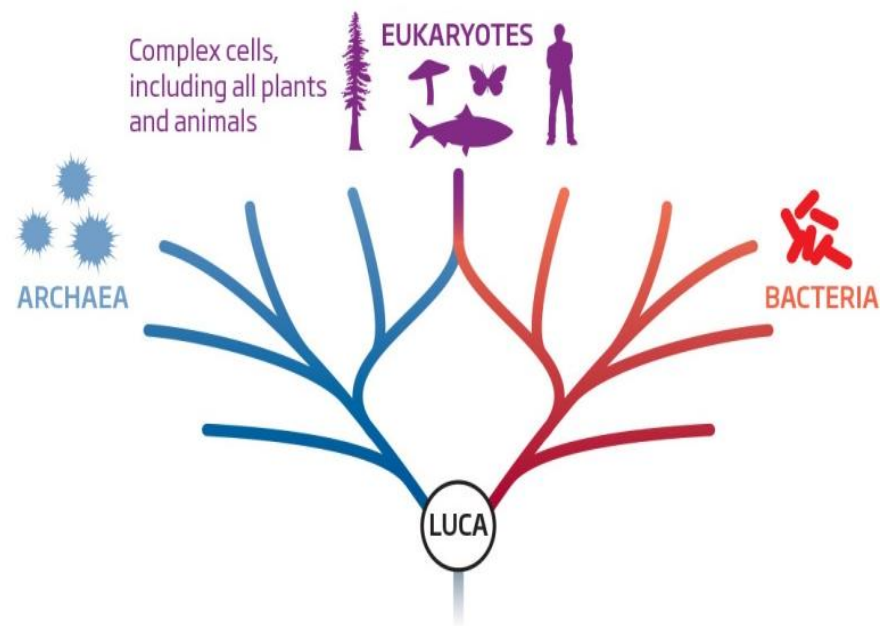


Fig.4 Mimivirus

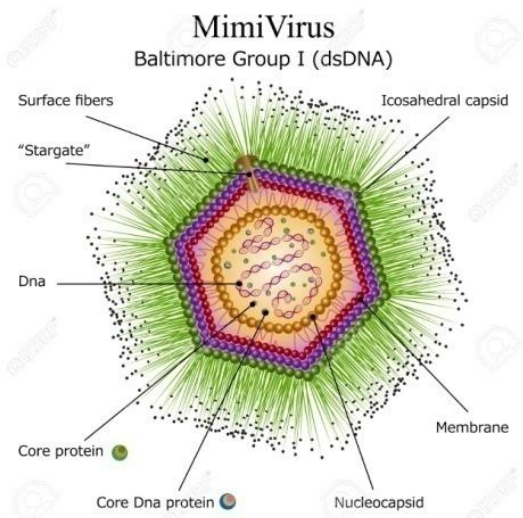

Fig.5 Sputnik Virion

Fig.6 Mamavirus
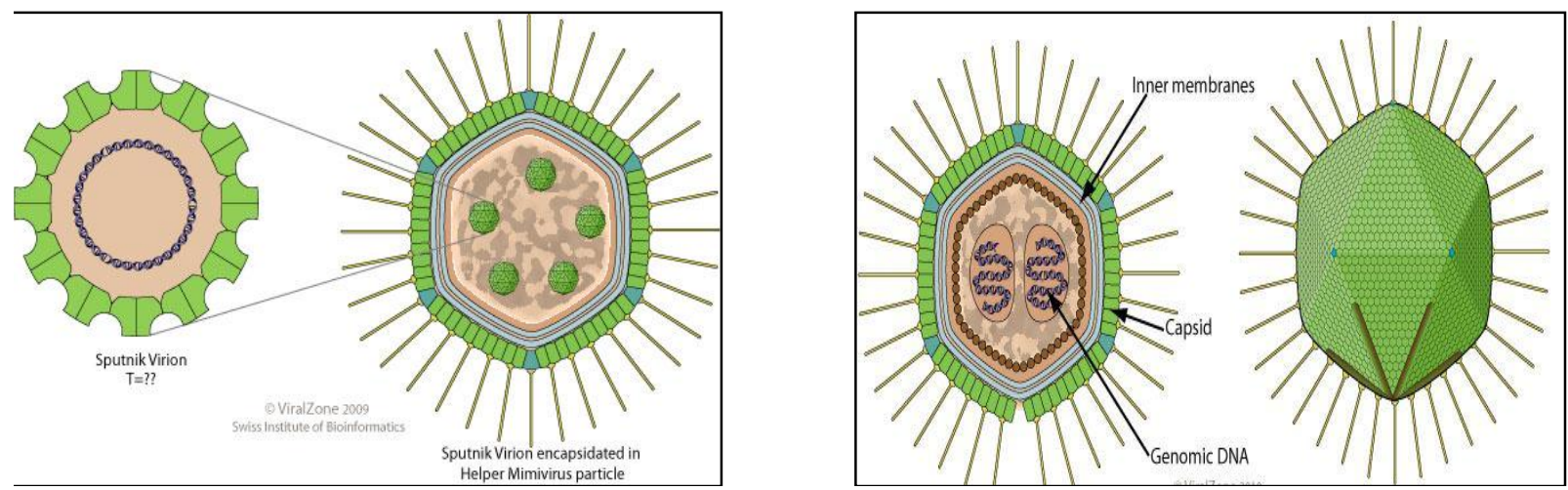

Fig.7 Protein folding

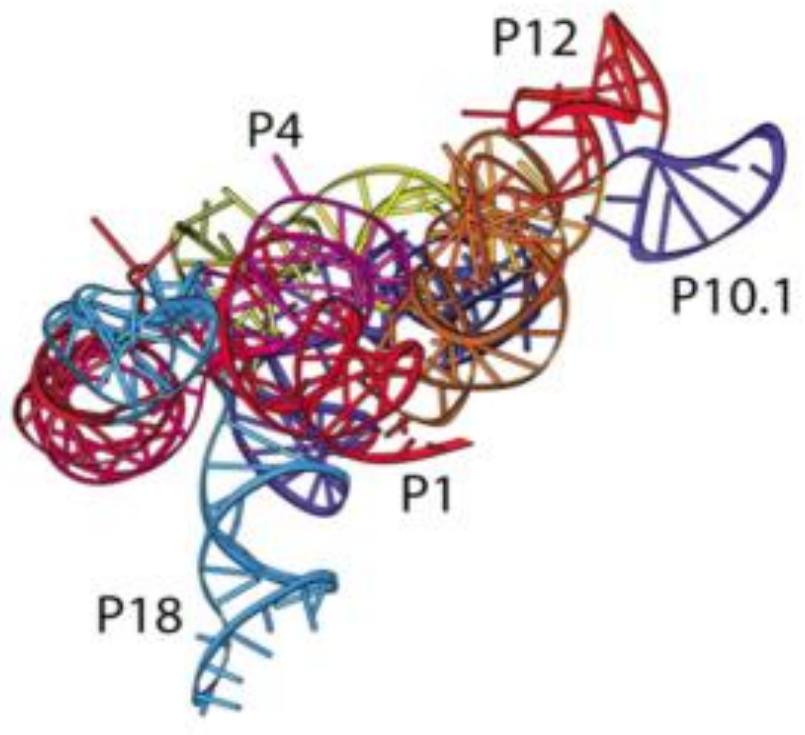


Fig.8 Ribosome - Capsid organisms

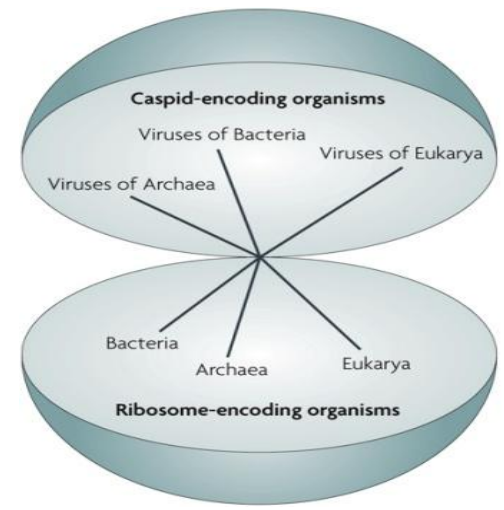

But the hallmark genes of viruses have no obvious ancestors in cellular forms (Eugene V Koonin et al., 2015). Another theory suggests that viruses may be evolved from bits of DNA/RNA that escaped from large genes (Transposons).

As the widely accepted theory suggests both virus and modern bacteria had a common ancestors. Viruses can be considered as a living organism but of a different fourth domain.

\section{Viruses get sick?}

Considering virus whether alive or dead remained dormant for few years until the recent discovery of GIANT VIRUS called the MIMIVIRUS (Fig. 4) (Mimicking Microbe) in 2003 by Didier Raoult et al., Mimivirus also contained certain eukaryotic genes. Later another giant virus called Mamavirus which was discovered in a cooling tower of Bradford, UK. As reported by La scola et al., in 2008, this giant virus contained 900 protein coding genes which is higher than the previously known Acanthamoeba polyphaga Mimivirus.

This virus was nearly the size of a bacterial cell and it is known to attack its host, the Amoeba.
But the real root cause of the debate is that another small sub-viral agent called SPUTNIK (Sputnik-3) invades the Mimivirus and thereby disrupting the replication cycle of Mimivirus ultimately making it Sick. Sputnik is the first known virophage (Fig. 5).

Thus Jean Michel Claverie concludes that "There's no doubt, this is a living organism, the fact that it can get sick makes it more alive" (Pearson H, 2008). Sputnik discovery also suggests the fact that these viruses could be having major effects on the ocean's nutrient cycle.

After Mimi and Mama virus (Fig. 6) even larger viruses have been discovered. In 2011, largest known then virus called the Megavirus Chilensis was found and in the year 2013, the largest virus (Genomewise) known upto date called the Pandora virus was discovered by Prof Jean Michel Claverie and Dr Chantal Abergel et al., 2013. The genome of Pandora virus was twice as large as Mimi and Mama viruses.

\section{Is protein folding the answer?}

A new study uses protein folds as evidences to declare that viruses are living. These protein folds do serve as a marker for evolutionary studies. The researchers Gustavo 
Caetano Anolles et al., (Fig. 7) analyzed all of the known folds in 5080 organisms representing all branches of the tree of life, including 3460 viruses. They identified 442 protein folds were shared proves that virus do exhibit properties that cells have. Thus Caetano-Anolles concludes that "Viruses now merit a place in by Gustavo the tree of life. Obviously, there is much more to viruses than once thought".

The tiny particle that remained as an enigma for more than a century and still exists as an enigma continuously baffling minds of mankind by its contradictory characters, is surely a biological puzzle. But various scientists, virologists have stated their views and endlessly debating whether viruses are alive or dead. John Mattik's views on this particle are "People say viruses aren't free living but that's a philosophical question- Are we free living? Life is an interconnected system". What was once considered as a mere box of biochemicals till the giant virus discovery? This historic encounter has rekindled the debate and it has also led to the fact that "It suggests there are other representatives of this viral family out there in the environment", Koonin says, Viruses are ultimately polyphyletic.

In contrary, if a particle containing nucleic acid, protein, lipid and carbohydrates are considered as a living entity, then scientists put forth the point that DNA/RNA must also be considered as a living thing.

Thereby considering all the above traits, first of all our current views and definitions of life is very constricted. Thus the root is faulty. Our view must be a bit more elaborate, as virologist Didier Raoult suggests to define, life into 2 major forms/groups:

Ribosome encoding organisms (Archae, Bacteria, Eukarya) (Fig. 8).
Capsid encoding organism (Virus).

Or, we can accept viruses to be the fourth domain of life. Though they are at the edge of life, drawing a fine line between natural and synthetic, viruses surely exhibit some key characteristics of living. Although they are still regarded as particles, agents and nonliving they indeed occupy a major aspect in the web of life.

Thus I surmise by claiming that these viral entities clearly reside as an element in this realm of life.

\section{Acknowledgement}

The author expresses her sincere thanks to the staff of her college for their valuable guidance and support. She further extends her thanks to her friends for their encouragement and to her family members for their support in completing this review article.

\section{References}

Adam, "Eclipse Period" - Medilexicon, 1959. Are viruses Dead or Alive? - Khan Academy. Eugene V Koonin, Valerian V Doljia, Mart Krupovic, Origins and evolution of viruses of eukaryotes: The ultimate modularity, Elsevier, May 2015, Vol 479-480, pp. 2-25.

Grennan Milliken, Are virus alive? New evidence says yes- Popular Science Magazine, Sep. 2015.

Gustavo Caetano-Anolles, Arshan Nasir, A phylogenomic data-driven exploration of viral origins and evolution, Science Advances, Sep. 2015.

Jake port, "Why are viruses considered to be non-living?"- Cosmos magazine, Sep 2017.

Jean Michel Claverie, Chantal Abergel, Nadege Philippe, Matthieu Legendre, Coute Gabriel, Poirot Yohann, Lescot 
Oliver, Arslan Magali, Virginie Seltzer, Lionel Bertaux, Christophe Bruley, Jerome Garin, Pandoraviruses: Amoeba viruses with Genomes up to $2.5 \mathrm{MB}$ reaching that of parasitic Eukaryotes, Science, 2013, vol 341, pp $281-286$.

Lecoq H, "Discovery of first virus - The Tobacco Mosaic virus: 1892 or 1898?" C R Acad Science III 2001 Oct324(10), pp 929-933.

Luis P. Villarreal, "Are viruses Alive?" Scientific American, Dec 2004.

Patrick Fortterre, "Defining Life: The virus viewpoint", Springer, Apr 2010, 40(2), 151-160 pp.
Pearson H, 'Virophage' suggest viruses are alive- Nature Aug 2008.

Sara Reardon, Giant virus discovery sparks debate over tree of life, Nature Apr 2009.

The Viral Life Cycle - Microbiology (Lumen Learning)

Venkataraman Prasad, B.V., and Michel F Schmid, "Principles of virus structural organization" - Advances in Exp Med Biol-2012, 726: 17-47.

Viviane Ritcher, What came first, cells or viruses? - Cosmos magazine, Oct 2015.

Wikipedia - Life.

\section{How to cite this article:}

Shwetha, S. 2018. Virus - The Biological Brain Twister. Int.J.Curr.Microbiol.App.Sci. 7(05): 54-61. doi: https://doi.org/10.20546/ijcmas.2018.705.008 